\title{
Nucleation in binary polymer blends: Effects of adding diblock copolymers
}

\author{
Jiafang Wang, Hongdong Zhang, and Feng Qiu \\ Department of Macromolecular Science, Key Lab of Molecular Engineering of Polymers, \\ Ministry of Education of China, Fudan University, Shanghai 200433, China \\ Zhen-Gang Wanga) \\ Division of Chemistry and Chemical Engineering, California Institute of Technology, Pasadena, \\ California 91125 \\ Yuliang Yang ${ }^{\text {b) }}$ \\ Department of Macromolecular Science, Key Lab of Molecular Engineering of Polymers, \\ Ministry of Education of China, Fudan University, Shanghai 200433, China
}

(Received 24 January 2003; accepted 20 February 2003)

\begin{abstract}
The effects of adding $\mathrm{AB}$ diblock copolymers to $\mathrm{A} / \mathrm{B}$ binary blends on the structure and thermodynamics of critical nuclei are studied using the self-consistent field theory. At a fixed ratio of the amount of the two homopolymers, depending on the degree of polymerization and composition of the diblocks, their addition to the blends can either increase or decrease the nucleation free energy barrier relative to the pure A/B blends. The qualitative trend can be deduced from the shift in the coexistence boundary and the spinodal. The distribution of diblock copolymers in critical nuclei depends on the composition of the diblocks and the quench depth. Near the coexistence, symmetric diblocks exhibit surfactant behavior, being highly concentrated on the interface of the critical nuclei. Near the spinodal, they act more like co-solvent with a relatively uniform distribution. (C) 2003 American Institute of Physics. [DOI: 10.1063/1.1566941]
\end{abstract}

\section{INTRODUCTION}

Recently, the self-consistent field theory (SCFT) was applied to the study of homogeneous nucleation in an $\mathrm{A} / \mathrm{B}$ binary polymer blend. ${ }^{1}$ Within the framework of mean-field theory, the SCFT avoids some common approximations that are often made in calculating the properties of the critical nuclei, such as the capillary approximation near the coexistence and the square-gradient approximation near the spinodal, and thus allows the entire range of the metastable region of the phase diagram to be studied using a single theoretical framework. Furthermore, by a quantitative evaluation of the nucleation free energy barrier near the spinodal, the SCFT provides a consistency criterion for the validity of the mean-field approximation as well as predicts the onset of crossover in the phase separation kinetics from nucleation to spinodal decomposition.

In this paper, we use SCFT to address the effects of $\mathrm{AB}$ diblock copolymers on nucleation in $\mathrm{A} / \mathrm{B}$ homopolymer blends. Specifically, we wish to answer the following question: Given a fixed ratio of the amount of A and B homopolymers, does the addition of a small amount of $A B$ diblock copolymers increase or decrease the nucleation free energy barrier? The answer to this question is not obvious, as there are several competing factors at play in governing the nucleation behavior of the system. On one hand, it is expected that

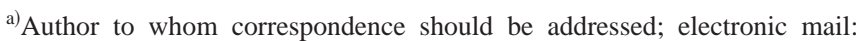
zgw@cheme.caltech.edu

b) Author to whom correspondence should be addressed; electronic mail: ylyang@srcap.stc.sh.cn
}

being amphiphilic molecules, the $\mathrm{AB}$ diblocks will accumulate at the interface of the nuclei, thus lowering the interfacial free energy cost for the formation of the droplets, but this occurs at the expense of the translational entropy of the diblocks. On the other hand, the addition of $\mathrm{AB}$ diblocks to A/B homopolymer blends shifts the coexistence and the spinodal, thus changing the thermodynamic driving force for nucleation, with the sign of the shift depending on the degrees of polymerization and the composition of the polymers.

Our interest in this problem is motivated by three considerations. First, multicomponent homopolymer and block copolymer blends are systems of wide industrial applications and theoretical interest. ${ }^{2-6}$ The $\mathrm{A} / \mathrm{B} / \mathrm{AB}$ ternary system serves as the simplest model system for understanding the complex phase behaviors in such blends. The roles of $\mathrm{AB}$ diblock copolymers on the equilibrium, bulk properties in A/B blends are relatively well understood: The diblocks can either act as surfactants to lower the interfacial tension by adsorbing at the A/B interface, or serve as co-solvent and distribute uniformly throughout the blends to improve their compatibility, or phase separate into a new copolymer-rich phase. ${ }^{6-10}$ However, there has been no theoretical work that elucidates the roles of the diblocks in the nucleation event that leads to phase separation. Therefore, our study will nicely complement previous theoretical work on the equilibrium, bulk properties, and will provide the theoretical basis for controlling the nucleation behavior in $\mathrm{A} / \mathrm{B}$ blends by the addition of the $\mathrm{AB}$ diblock copolymers.

Second, several experiments by Balsara and co-workers 
aimed at probing the nucleation in A/B binary homopolymer blends involved adding an $\mathrm{AB}$ diblock copolymer in order to lower the demixing temperature and thus control the distance to the phase boundary without signficantly affecting the time scales for molecular motion. ${ }^{11,12}$ Their work showed that increasing the amount of diblock copolymers can either switch the mechanism of phase separation from spinodal decomposition to nucleation ${ }^{11}$ or decrease the rate of nucleation. ${ }^{12}$ These authors also argued, based on the theoretical work mentioned earlier, that in the temperature and composition ranges of the experiments, the block copolymers primarily act as co-solvent distributing themselves uniformly in the blends at all (early) stages of the phase separation. However, the validity of such arguments is a priori not clear without directly studying the problem of nucleation, because the block copolymers may well play a different role in the inhomogeneous nuclei from that in the uniform, equilibrium bulk state or from that near the equilbirum interface of the phaseseparated blends. For example, while the equilibrium interfacial width decreases with increasing Flory-Huggins parameter $\chi$, the interfacial region of the critical nuclei broadens as $\chi$ increases from the coexistence to the spinodal. Our study will allow us to address both the distribution of the diblock copolymers and their effects on nucleation barrier.

Finally, as amphiphilic molecules, AB diblock copolymers share many common features with other surfactant systems. Density functional theory has been applied to study gas-to-liquid nucleation in the presence of amphiphilic molecules. ${ }^{13}$ Nucleation of micelles has also been studied using both density functional theory ${ }^{14}$ and SCFT. ${ }^{15}$ While these studies provide useful insights into nucleation involving amphiphilic molecules, it is desirable to address directly the effects of surfactant molecules on nucleation in liquidliquid phase separation.

The rest of this paper is organized as follows. In Sec. II, we briefly describe the SCFT and the numerical method applied to the calculation of critical nuclei in an $\mathrm{A} / \mathrm{B} / \mathrm{AB}$ blend. In order to make reference to the bulk phase diagram of the system, we also provide a brief review of the relevant results for the coexistence and the spinodal of the system. The main results are presented and discussed in Sec. III. We first focus on symmetric diblock copolymers added to binary blends of $\mathrm{A}$ and $\mathrm{B}$ homopolymers having equal degree of polymerization in order to highlight the main effects without the complications due to the introduction of extra parameters. The effects of asymmetry of the diblocks are then presented separately. We conclude in Sec. IV with a summary of the main results of our findings.

\section{THEORY AND METHODOLOGY}

\section{A. Self-consistent field theory}

We consider an incompressible ternary blend of homopolymers A and B (hereafter designated as HA and HB, respectively) and a diblock copolymer $\mathrm{AB}$ (designated as $\mathrm{AB})$, with degrees of polymerization $N_{\kappa}(\kappa=\mathrm{HA}, \mathrm{HB}, \mathrm{AB})$. The polymers are modeled as Gaussian chains, and for simplicity we assume the same monomeric volume $v$ and Kuhn length $b$ for all the polymer species. We choose units for the volume so that $v=1$. For studying nucleation in a metastable bulk phase, it is convenient to work with the grand canonical ensemble with chemical potentials $\mu_{\kappa}$. Because the chemical potentials of the components in an incompressible mixture are not all independent, we set $\mu_{\mathrm{AB}}=0$. The derivation of the SCFT is similar to that given in Refs. 16 and 17 and will not be repeated here. The grand potential can be written as

$$
\begin{aligned}
& \beta G\left[\phi_{\mathrm{A}}, \phi_{\mathrm{B}}, \omega_{\mathrm{A}}, \omega_{\mathrm{B}}, \xi\right] \\
& =\int d r\left[\chi \phi_{\mathrm{A}}(r) \phi_{\mathrm{B}}(r)-\omega_{\mathrm{A}}(r) \phi_{\mathrm{A}}(r)-\omega_{\mathrm{B}}(r) \phi_{\mathrm{B}}(r)\right] \\
& \quad-\frac{\exp \left(\beta \mu_{\mathrm{HA}} N_{\mathrm{HA}}\right)}{N_{\mathrm{HA}}} Q_{\mathrm{HA}}\left[\omega_{\mathrm{A}}\right] \\
& \quad-\frac{\exp \left(\beta \mu_{\mathrm{HB}} N_{\mathrm{HB}}\right)}{N_{\mathrm{HB}}} Q_{\mathrm{HB}}\left[\omega_{\mathrm{B}}\right]-\frac{1}{N_{\mathrm{AB}}} Q_{\mathrm{AB}}\left[\omega_{\mathrm{A}}, \omega_{\mathrm{B}}\right] \\
& \quad+\int d r \xi(r)\left[\phi_{\mathrm{A}}(r)+\phi_{\mathrm{B}}(r)-1\right],
\end{aligned}
$$

where $\beta=1 /\left(k_{\mathrm{B}} T\right)$ and $\chi$ is Flory-Huggins interaction parameter, $\phi_{\mathrm{A}}$ and $\phi_{\mathrm{B}}$ are the total monomer volume fraction of $\mathrm{A}$ and $\mathrm{B}$, respectively, with contributions from both the homopolymer and diblock copolymer, $\omega_{\alpha}(\alpha=\mathrm{A}, \mathrm{B})$ is the molecular self-consistent field conjugate to $\phi_{\alpha}$, and $\xi$ is an effective pressure field to ensure the incompressibility constraint. Note that the cube of the thermal de Broglie wavelength is replaced by the volume of the chain $N_{\kappa} v$; this simply leads to inconsequential shift in the chemical potential.

In Eq. (1), $Q_{\kappa}$ is the single chain partition function of chain $\kappa$ in the presence of external fields $\omega_{\mathrm{A}}$ and $\omega_{\mathrm{B}}$ :

$$
Q_{\kappa}\left[\omega_{\mathrm{A}}, \omega_{\mathrm{B}}\right]=\int d r q_{\kappa}\left(r, N_{\kappa}\right), \quad \kappa: \mathrm{HA}, \mathrm{HB}, \mathrm{AB},
$$

where the end-segment distribution function $q_{\kappa}\left(r, N_{\kappa}\right)$ is obtained from solving the modified diffusion equation

$$
\left[\frac{\partial}{\partial \tau}-\frac{b^{2}}{6} \nabla_{r}^{2}+\omega_{\kappa}(r)\right] q_{\kappa}(r, \tau)=0, \quad \kappa: \mathrm{HA}, \mathrm{HB}
$$

for homopolymers, and

$$
\begin{aligned}
& {\left[\frac{\partial}{\partial \tau}-\frac{b^{2}}{6} \nabla_{r}^{2}+\omega_{\mathrm{A}}(r)\right] q_{\mathrm{AB}}(r, \tau)=0, \quad 0<\tau<f N_{\mathrm{AB}},} \\
& {\left[\frac{\partial}{\partial \tau}-\frac{b^{2}}{6} \nabla_{r}^{2}+\omega_{\mathrm{B}}(r)\right] q_{\mathrm{AB}}(r, \tau)=0, \quad f N_{\mathrm{AB}}<\tau<N_{\mathrm{AB}}}
\end{aligned}
$$

for the diblock copolymer, and $f$ is the A-block composition in the diblock. The initial conditions are $q_{\kappa}(r, 0)=1$. Because of the lack of inversion symmetry for the two blocks in the diblock copolymer $\mathrm{AB}$, it is necessary to introduce a conjugate end-segment distribution $q_{\mathrm{AB}}^{*}(r, \tau)$, which satisfies a similar modified diffusion equation to Eq. (4) with $\partial / \partial \tau$ multiplied by -1 and with initial condition $q_{\mathrm{AB}}^{*}\left(r, N_{\mathrm{AB}}\right)=1$.

The self-consistent field equations are obtained by a variational extremization of the grand potential with respect to $\phi_{\mathrm{A}}, \phi_{\mathrm{B}}, \omega_{\mathrm{A}}, \omega_{\mathrm{B}}, \xi$ respectively, which yields

$$
\begin{aligned}
& \omega_{\mathrm{B}}=\chi \phi_{\mathrm{A}}+\xi, \\
& \omega_{\mathrm{A}}=\chi \phi_{\mathrm{B}}+\xi,
\end{aligned}
$$




$$
\begin{aligned}
\phi_{\mathrm{A}}= & \frac{e^{\beta \mu_{\mathrm{HA}} N_{\mathrm{HA}}}}{N_{\mathrm{HA}}} \int_{0}^{N_{\mathrm{HA}}} d \tau q_{\mathrm{HA}}(r, \tau) q_{\mathrm{HA}}\left(r, N_{\mathrm{HA}}-\tau\right) \\
& +\frac{1}{N_{\mathrm{AB}}} \int_{0}^{f N_{\mathrm{AB}}} d \tau q_{\mathrm{AB}}(r, \tau) q_{\mathrm{AB}}^{*}(r, \tau), \\
\phi_{\mathrm{B}}= & \frac{e^{\beta \mu_{\mathrm{HB}} N_{\mathrm{HB}}}}{N_{\mathrm{HB}}} \int_{0}^{N_{\mathrm{HB}}} d \tau q_{\mathrm{HB}}(r, \tau) q_{\mathrm{HB}}\left(r, N_{\mathrm{HB}}-\tau\right) \\
& +\frac{1}{N_{\mathrm{AB}}} \int_{f N_{\mathrm{AB}}}^{N_{\mathrm{AB}}} d \tau q_{\mathrm{AB}}(r, \tau) q_{\mathrm{AB}}^{*}(r, \tau), \\
\phi_{\mathrm{A}}+ & \phi_{\mathrm{B}}=1 .
\end{aligned}
$$

In order to calculate the free energy barrier for the formation of the critical nucleus, we need the grand potential of the metastable homogeneous state. For the metastable homogeneous state, the self-consistent field equation set can be solved readily. The molecular field can be calculated as

$$
\begin{aligned}
& \omega_{\mathrm{A}}^{0}=-\frac{\ln \left(\phi_{\mathrm{AB}}^{0}\right)}{N_{\mathrm{AB}}}+(1-f) \chi\left[1-2\left(\phi_{\mathrm{HA}}^{0}+f \phi_{\mathrm{AB}}^{0}\right)\right], \\
& \omega_{\mathrm{B}}^{0}=-\frac{\ln \left(\phi_{\mathrm{AB}}^{0}\right)}{N_{\mathrm{AB}}}-f \chi\left[1-2\left(\phi_{\mathrm{HA}}^{0}+f \phi_{\mathrm{AB}}^{0}\right)\right]
\end{aligned}
$$

and the chemical potential can be written as

$$
\begin{aligned}
\beta \mu_{\mathrm{HA}}= & \frac{\ln \phi_{\mathrm{HA}}^{0}}{N_{\mathrm{HA}}}-\frac{\ln \phi_{\mathrm{AB}}^{0}}{N_{\mathrm{AB}}}+(1-f) \chi\left[1-2\left(\phi_{\mathrm{HA}}^{0}\right.\right. \\
& \left.\left.+f \phi_{\mathrm{AB}}^{0}\right)\right] \\
\beta \mu_{\mathrm{HB}}= & \frac{\ln \phi_{\mathrm{HB}}^{0}}{N_{\mathrm{HB}}}-\frac{\ln \left(\phi_{\mathrm{AB}}^{0}\right)}{N_{\mathrm{AB}}}-f \chi\left[1-2\left(\phi_{\mathrm{HA}}^{0}+f \phi_{\mathrm{AB}}^{0}\right)\right]
\end{aligned}
$$

Therefore, the grand potential density is

$$
\begin{aligned}
g^{0}= & \chi\left(\phi_{\mathrm{HA}}^{0}+f \phi_{\mathrm{AB}}^{0}\right)^{2}+\chi f\left[1-2\left(\phi_{\mathrm{HA}}^{0}+f \phi_{\mathrm{AB}}^{0}\right)\right] \\
& +\frac{\ln \phi_{\mathrm{AB}}^{0}}{N_{\mathrm{AB}}}-\frac{\phi_{\mathrm{HA}}^{0}}{N_{\mathrm{HA}}}-\frac{\phi_{\mathrm{HB}}^{0}}{N_{\mathrm{HB}}}-\frac{\phi_{\mathrm{AB}}^{0}}{N_{\mathrm{AB}}} .
\end{aligned}
$$

Subtracting the grand potential for the uniform metastable phase from that for the critical nucleus, we obtain the reversible work of formation, or the free energy barrier for nucleation as

$$
\begin{aligned}
\Delta F^{*}= & G^{*}\left\{\phi_{\mathrm{A}}^{*}, \phi_{\mathrm{B}}^{*}, \omega_{\mathrm{A}}^{*}, \omega_{\mathrm{B}}^{*}, \xi^{*}\right\}-G^{0} \\
= & 4 \pi \int_{0}^{\infty} r^{2} d r\left\{\chi \phi_{\mathrm{A}}^{*} \phi_{\mathrm{B}}^{*}-\chi \phi_{\mathrm{A}}^{0} \phi_{\mathrm{B}}^{0}\right. \\
& -\sum_{\alpha=\mathrm{A}, \mathrm{B}}\left[\omega_{\alpha}^{*} \phi_{\alpha}^{*}-\omega_{\alpha}^{0} \phi_{\alpha}^{0}\right] \\
& \left.-\sum_{\kappa=\mathrm{HA}, \mathrm{HB}, \mathrm{AB}}\left[\frac{\exp \left(\beta \mu_{\kappa} N_{\kappa}\right)}{N_{\kappa}} q_{\kappa}^{*}\left(r, N_{\kappa}\right)-\frac{\phi_{\kappa}^{0}}{N_{\kappa}}\right]\right\},
\end{aligned}
$$

where $\phi_{\alpha}^{*}, \omega_{\alpha}^{*}(\alpha=\mathrm{A}, \mathrm{B})$ and $\xi^{*}$ are the solutions to the self-consistent field equations for the critical nucleus. For simplicity of notation, we will omit the superscript asterisk

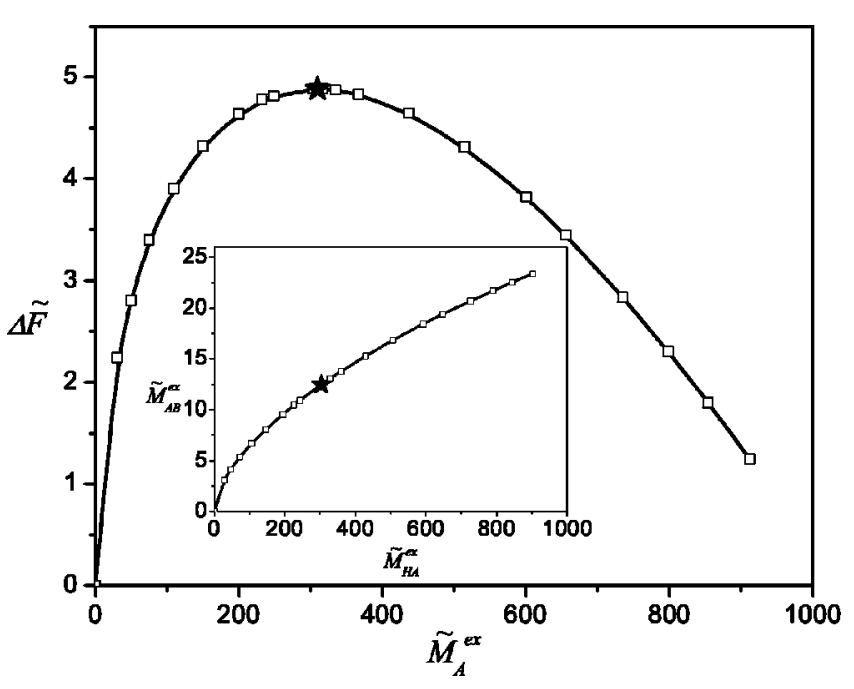

FIG. 1. Free energy of formation of the nuclei as function of the material excess of the total A segments for $\widetilde{\phi}_{\mathrm{HA}}^{0}=0.16, \phi_{\mathrm{AB}}^{0}=0.1, f=0.5, \alpha_{\mathrm{AB}}$ $=1.0$, and $\tilde{\chi}=1.38$. The maximum at $\widetilde{M}_{\mathrm{A}}^{\mathrm{ex}} \approx 310$ marked by a star corresponds to the critical nucleus. The inset shows the relationship between the material excess from the $\mathrm{AB}$ diblock and that from the homopolymer HA on the nucleation path.

in these quantities. We define the material excess $M_{\mathrm{A}}^{\mathrm{ex}}$ and the radius of the critical nucleus $R$ using the total density profile of the A segments $\phi_{\mathrm{A}}: M_{\mathrm{A}}^{\mathrm{ex}} \equiv 4 \pi \int_{0}^{\infty} r^{2} d r\left[\phi_{\mathrm{A}}(r)-\phi_{\mathrm{A}}^{0}\right]$ and

$$
R \equiv \frac{4 \pi}{M_{\mathrm{A}}^{\mathrm{ex}}} \int_{0}^{\infty} r^{3} d r\left[\phi_{\mathrm{A}}(r)-\phi_{\mathrm{A}}^{0}\right] .
$$

Similarly, $M_{\mathrm{HA}}^{\mathrm{ex}}$ and $M_{\mathrm{AB}}^{\mathrm{ex}}$ are defined using the density profiles of the homopolymer HA and the diblock AB.

\section{B. Identifying the critical nucleus}

Because the critical nucleus corresponds to a free energy maximum in the material excess of the nucleating species, straightforward application of the SCFT will not yield the desired solution. To capture the rare concentration fluctuations leading to nucleation, the calculation must be constrained with respect to the material excess. Here we follow a similar scheme to that in Ref. 1 in the pure HA/HB binary blend. However, because there are two independent concentration variables in the current case as opposed to the pure $\mathrm{HA} / \mathrm{HB}$ binary blend, the full free energy surface should involve both $\mathrm{HA}$ and $\mathrm{AB}$. By constraining only the overall material excess of the A monomers $M_{\mathrm{A}}^{\mathrm{ex}}$, the free energy is thus minimized with respect to the partitioning of the overall $M_{\mathrm{A}}^{\mathrm{ex}}$ into contributions coming from the homopolymer HA $M_{\mathrm{HA}}^{\mathrm{ex}}$ and from the $\mathrm{AB}$ diblocks $M_{\mathrm{AB}}^{\mathrm{ex}}$. This allows us to reduce the two-dimensional reaction coordinate into a onedimensional one. The $M_{\mathrm{HA}}^{\mathrm{ex}}$ versus $M_{\mathrm{AB}}^{\mathrm{ex}}$ plot as we increase $M_{\mathrm{A}}^{\mathrm{ex}}$, therefore, corresponds exactly to the nucleation path in these two variables. A typical one-dimensional free energy surface is shown in Fig. 1, with the inset showing the nucleation path in the two order parameters $M_{\mathrm{HA}}^{\mathrm{ex}}$ and $M_{\mathrm{AB}}^{\mathrm{ex}}$ (The dimensionless properties $\Delta \widetilde{F}, \tilde{M}_{\mathrm{A}}^{\mathrm{ex}}, \tilde{M}_{\mathrm{HA}}^{\mathrm{ex}}$, and $\tilde{M}_{\mathrm{AB}}^{\mathrm{ex}}$ defined in Sec. III are used in Fig. 1). 


\section{Phase diagram}

In order to specify the thermodynamic state of the homogeneous metastable phase, we provide a brief review of the relevant part of the phase diagram for our ternary system. The full phase diagram for the $\mathrm{HA} / \mathrm{HB} / \mathrm{AB}$ ternary blend is quite complicated. ${ }^{5-7,17,18} \mathrm{~A}$ large amount of diblock copolymer would lead to microphase separation. Because we are interested in nucleation for macrophase separation, we thus limit our consideration to block copolymer concentrations below the Lifshitz point. ${ }^{6}$ From the solution to the selfconsistent field equations for the homogeneous state, we obtain the free energy density of mixing for the $\mathrm{HA} / \mathrm{HB} / \mathrm{AB}$ ternary blend:

$$
\begin{aligned}
\Delta f_{\text {mix }}= & \frac{\phi_{\mathrm{AB}}^{0} \ln \left(\phi_{\mathrm{AB}}^{0}\right)}{N_{\mathrm{AB}}}+\frac{\phi_{\mathrm{HB}}^{0} \ln \left(\phi_{\mathrm{HB}}^{0}\right)}{N_{\mathrm{HB}}}+\frac{\phi_{\mathrm{HA}}^{0} \ln \left(\phi_{\mathrm{HA}}^{0}\right)}{N_{\mathrm{HA}}} \\
& +\chi\left(\phi_{\mathrm{HA}}^{0}+f \phi_{\mathrm{AB}}^{0}\right)\left[\phi_{\mathrm{HB}}^{0}+(1-f) \phi_{\mathrm{AB}}^{0}\right] \\
& -\phi_{\mathrm{AB}}^{0} \chi f(1-f) .
\end{aligned}
$$

For a given overall composition, the coexistence between the HA-rich and HB-rich phases is determined by the equality of chemical potential of species $\mathrm{HA}$ and $\mathrm{HB}$, respectively, in both phases, and the equality of the grand potential of the two phases. In general these conditions lead to a set of transcendental equations that can only be solved numerically.

The spinodal curve (the limit of metastability of the homogeneous phase) is determined by ${ }^{18}$

$$
\left|\begin{array}{ll}
f_{\text {HAHA }} & f_{\text {HAHB }} \\
f_{\text {HBHA }} & f_{\text {HBHB }}
\end{array}\right|=0
$$

with

$f_{I J}=\left(\frac{\partial}{\partial \phi_{I}}-\frac{\partial}{\partial \phi_{\mathrm{AB}}}\right)\left(\frac{\partial}{\partial \phi_{J}}-\frac{\partial}{\partial \phi_{\mathrm{AB}}}\right) \Delta f_{\mathrm{mix}}, \quad I, J=\mathrm{HA}, \mathrm{HB}$.

The condition (17) leads to an analytical expression for the value of the Flory-Huggins parameter at the spinodal:

$$
\chi_{\mathrm{sp}}=\frac{N_{\mathrm{AB}} \phi_{\mathrm{AB}}^{0}+N_{\mathrm{HA}} \phi_{\mathrm{HA}}^{0}+N_{\mathrm{HB}} \phi_{\mathrm{HB}}^{0}}{2\left[f^{2} N_{\mathrm{AB}} \phi_{\mathrm{AB}}^{0} N_{\mathrm{HB}} \phi_{\mathrm{HB}}^{0}+(1-f)^{2} N_{\mathrm{HA}} \phi_{\mathrm{HA}}^{0} N_{\mathrm{AB}} \phi_{\mathrm{AB}}^{0}+N_{\mathrm{HA}} \phi_{\mathrm{HA}}^{0} N_{\mathrm{HB}} \phi_{\mathrm{HB}}^{0}\right]} .
$$

\section{RESULTS AND DISCUSSION}

In order to highlight the main effects of diblock copolymers on nucleation without introducing too many parameters, in this study we assume the homopolymers HA and HB to be of the same degree of polymerization $\left(N_{\mathrm{HA}}=N_{\mathrm{HB}}\right.$ $=N$ ). The chain length of the added AB block copolymers is rescaled as $\alpha_{\mathrm{AB}}=N_{\mathrm{AB}} / N$. The length, material excess, and free energy barrier are nondimensionized by defining

$$
\begin{aligned}
& \widetilde{r} \equiv \frac{r}{N^{1 / 2} b}, \\
& \widetilde{M}_{\kappa}^{\mathrm{ex}} \equiv \frac{M_{\kappa}^{\mathrm{ex}}}{N^{3 / 2} b^{3}}, \quad \kappa: \mathrm{A}, \mathrm{HA}, \mathrm{AB}, \\
& \Delta \widetilde{F}=\frac{\beta \Delta F}{N^{1 / 2}} \frac{v}{b^{3}}
\end{aligned}
$$

and we define a reduced $\tilde{\chi}$ using the critical value of $\chi, \chi_{c, b}$ for the binary HA/HB blends in the absence of diblock copolymers:

$$
\tilde{\chi}=\chi / \chi_{c, b} .
$$

To focus on the effects of adding a small amount of the $\mathrm{AB}$ block copolymers on nucleation in the binary blends $\mathrm{HA} / \mathrm{HB}$, we imagine a thought experiment in which the ratio of the amount of $\mathrm{HA}$ and $\mathrm{HB}$ is held constant while the diblocks are added. To this end, it is convenient to express the amount of the block copolymers by their volume fraction in the ternary blends $\phi_{\mathrm{AB}}^{0}$, and the ratio of the amount of the homopolymers by their volume fractions in the original binary blends; that is,

$$
\widetilde{\phi}_{\mathrm{HA}}^{0}=\frac{\phi_{\mathrm{HA}}^{0}}{1-\phi_{\mathrm{AB}}^{0}}
$$

and

$$
\widetilde{\phi}_{\mathrm{HB}}^{0}=\frac{\phi_{\mathrm{HB}}^{0}}{1-\phi_{\mathrm{AB}}^{0}} .
$$

Fixing the ratio of the amount of HA and HB corresponds to fixing $\widetilde{\phi}_{\mathrm{HA}}^{0}$ (or $\widetilde{\phi}_{\mathrm{HB}}^{0}$ since the two add up to one). In the following, we first present the results for symmetric diblock copolymers since this case illustrates most clearly and simply the effects of these molecules on nucleation in the HA/HB blends. We then discuss the effects of asymmetry of the diblocks. For all the results we present, the reduced volume fraction is set at $\widetilde{\phi}_{\mathrm{HA}}^{0}=0.16$ unless otherwise specified; we do not expect qualitatively new effects by changing $\widetilde{\phi}_{\mathrm{HA}}^{0}$.

\section{A. Symmetric diblock copolymers}

If the added $\mathrm{AB}$ block copolymers are symmetric in composition $(f=0.5)$, the coexistence condition and the spinodal condition can be written analytically as 


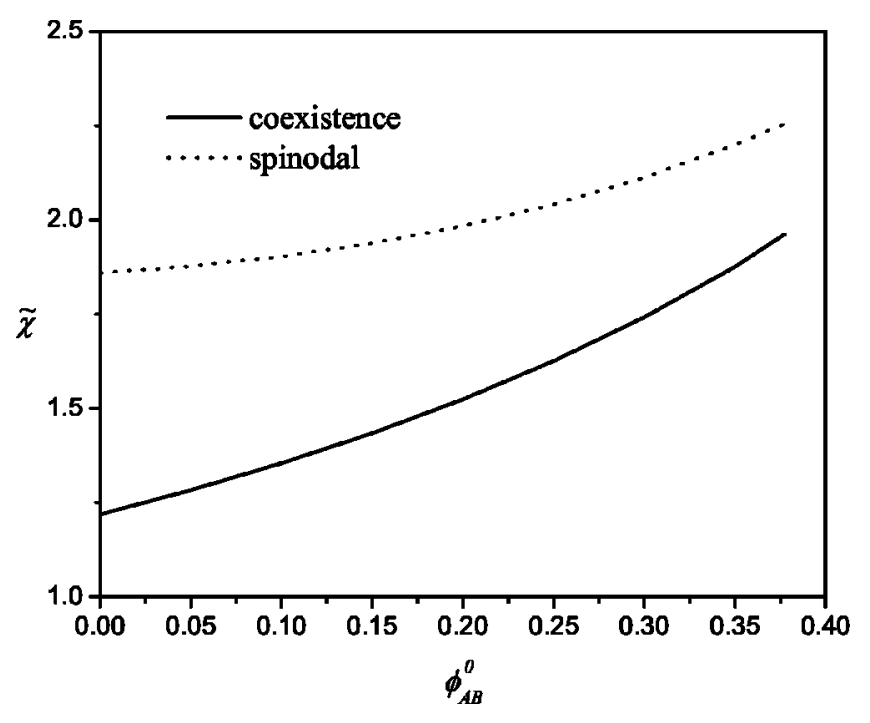

FIG. 2. Effect of the amount of added symmetric diblock copolymers of the same chain length as the homopolymers $\left(f=0.5, \alpha_{\mathrm{AB}}=1\right)$ on the coexistence and the spinodal in the $\mathrm{HA} / \mathrm{HB} / \mathrm{AB}$ ternary system at fixed $\widetilde{\phi}_{\mathrm{HA}}^{0}$ $=0.16$.

$$
\begin{aligned}
N \chi_{\mathrm{co}} & =-\frac{\ln \left(\frac{\phi_{\mathrm{HA}}^{0}}{1-\phi_{\mathrm{HA}}^{0}-\phi_{\mathrm{AB}}^{0}}\right)}{\left(1-2 \phi_{\mathrm{HA}}^{0}-\phi_{\mathrm{AB}}^{0}\right)} \\
& =-\frac{\ln \left(\frac{\widetilde{\phi}_{\mathrm{HA}}^{0}}{1-\widetilde{\phi}_{\mathrm{HA}}^{0}}\right)}{\left(1-2 \widetilde{\phi}_{\mathrm{HA}}^{0}\right)\left(1-\phi_{\mathrm{AB}}^{0}\right)}, \\
N \chi_{\mathrm{sp}} & =\frac{(1 / 2) \alpha_{\mathrm{AB}} \phi_{\mathrm{AB}}^{0}\left(1-\phi_{\mathrm{AB}}^{0}\right)+2\left(1-\phi_{\mathrm{AB}}^{0}\right)^{2} \widetilde{\phi}_{\mathrm{HA}}^{0} \widetilde{\phi}_{\mathrm{HB}}^{0}}{(21)} .
\end{aligned}
$$

In Fig. 2, we show the coexistence and the spinodal as a function of the amount of symmetric diblock copolymers added for the case of $\alpha_{\mathrm{AB}}=1$. Both the coexistence and the spinodal shift to higher values of the reduced $\tilde{\chi}$ with the addition of the diblocks. For a fixed amount of diblock copolymers $\phi_{\mathrm{AB}}^{0}$, the coexistence is independent of the degree of polymerization of the diblocks. However, the spinodal $\widetilde{\chi}_{\mathrm{sp}}$ decreases monotonically with increasing degrees of polymerization of the diblocks. This is shown in Fig. 3. We will make reference to these phase diagrams when we discuss the effects of diblock copolymers on nucleation.

Although adding the $\mathrm{AB}$ block copolymers shifts the binodal and the spinodal boundary of the binary blends HA/ $\mathrm{HB}$, the basic characteristics of the critical nuclei of macroscopic phase separation are similar to those in the pure binary blends $\mathrm{HA} / \mathrm{HB} .^{1}$ The work of critical nuclei formation decreases monotonically with the increase of $\tilde{\chi}$ from the binodal where it diverges, to the spinodal where it vanishes. The material excess and the radii of critical nuclei have their minima intermediate between the coexistence and the spin-

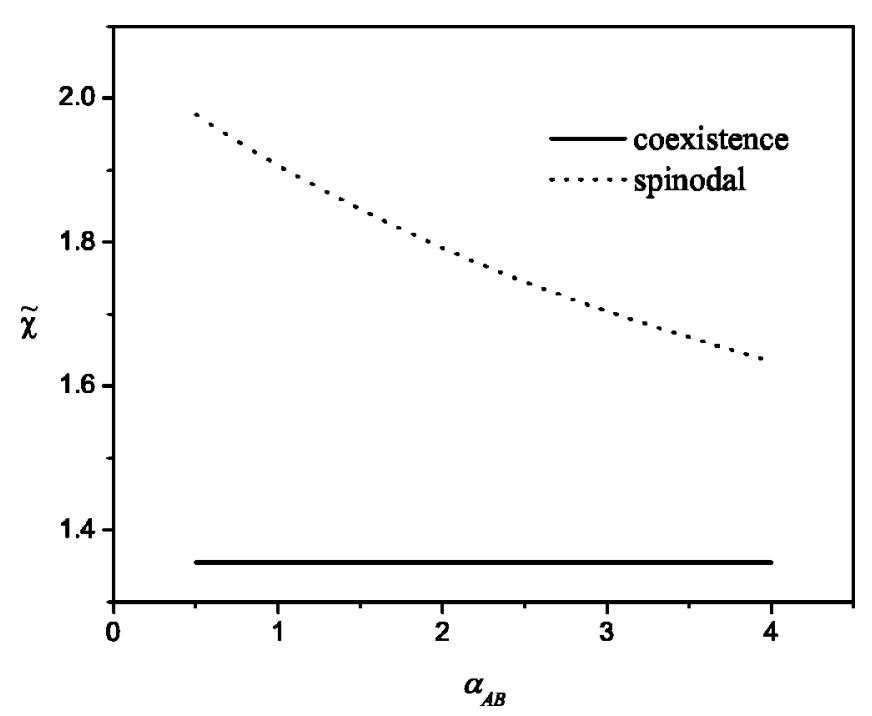

FIG. 3. Phase diagram showing the effect of the relative chain length of the symmetric diblock copolymers in the $\mathrm{HA} / \mathrm{HB} / \mathrm{AB}$ ternary blends at $\phi_{\mathrm{AB}}^{0}$ $=0.1$ and $\widetilde{\phi}_{\mathrm{HA}}^{0}=0.16$.

odal, and diverge in these two limits. Because these two properties have similar behaviors, only the radii of critical nuclei are discussed in this paper.

Even for diblock copolymer concentrations below the Lifshitz point, it is possible to form swollen micelles. Our preliminary calculations show that this can occur for increased concentration and/or length of the diblock copolymers. In this paper, we focus on nucleation leading to macroscopic phase separation and have chosen parameters such that micellization does not occur. Formation of swollen micelles will be investigated in a future study.

Near the coexistence, the critical nucleus is highly structured and has a sharp interface, much like the equilibrium interface between two coexisting phases. The nearly uniform density of the nucleating species inside the nucleus is very close to but slightly higher than the coexisting phase because of increased osmotic pressure due to curvature. Therefore, near the coexistence limit, the critical nucleus can be considered as a droplet of the incipient coexisting phase. As the immiscibility increases from the coexistence boundary toward the spinodal, the critical nucleus become less structured and the density variation becomes more gradual. This behavior is demonstrated in Fig. 4(a) where we show the overall density profile for A segments at several values of the reduced $\tilde{\chi}$ for $\phi_{\mathrm{AB}}^{0}=0.1$ and $\alpha_{\mathrm{AB}}=1$.

The behavior of the overall density of the A segments is strongly correlated with the distribution of the diblock copolymers. As shown in Fig. 4, near the binodal, the critical nucleus has a relatively sharp interface, with a strong enhancement of copolymer concentration in the interfacial region. The critical nucleus resembles emulsion droplet with the symmetric diblocks acting as surfactants. Upon increasing the quench depth to the spinodal, the interface of a critical nucleus becomes diffuse; the distribution of the diblocks becomes flat with only slight enrichment at the center of the nucleus. In the spinodal limit, the diblocks would distribute uniformly just like co-solvent. Therefore, the behavior of 

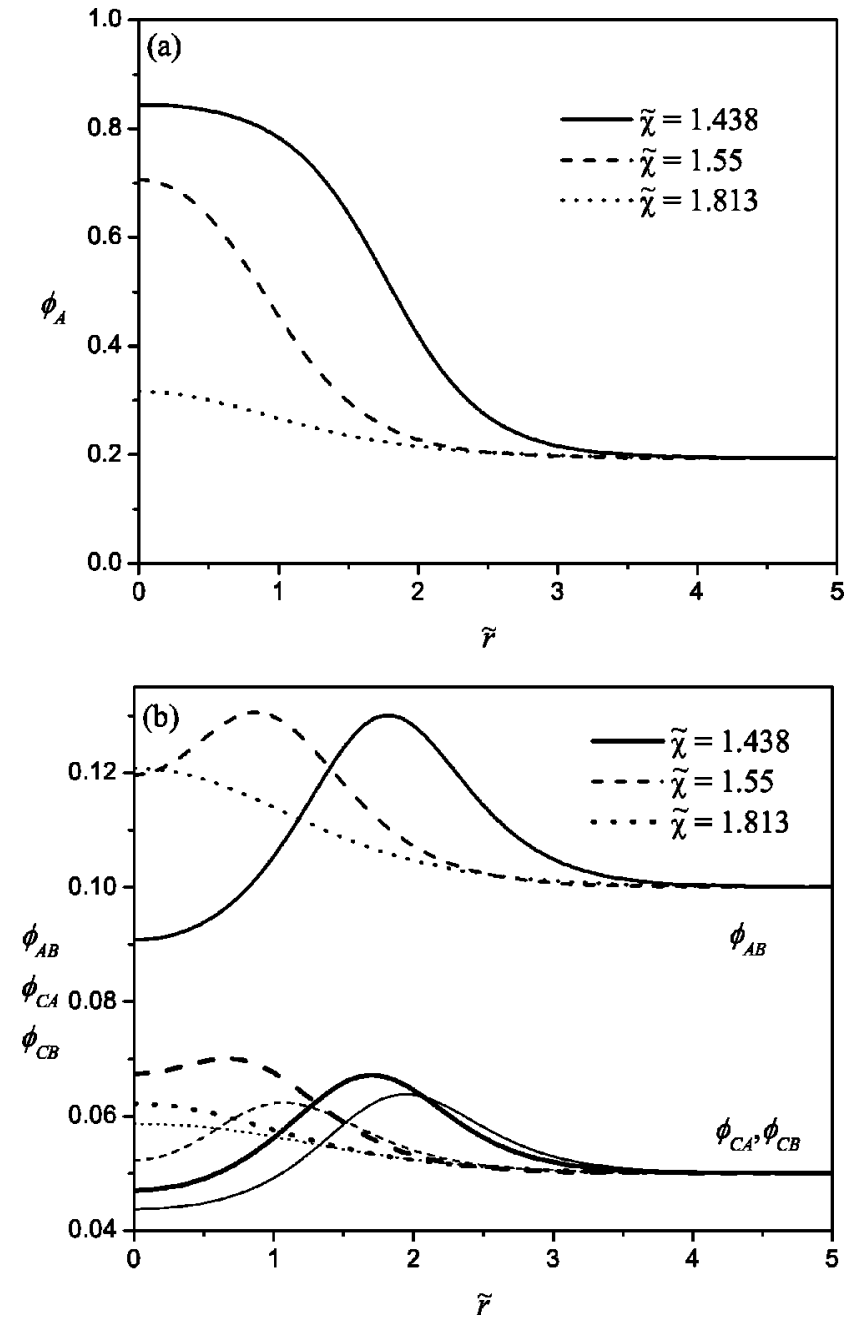

FIG. 4. Three representative density profiles for the critical nuclei with symmetric $\mathrm{AB}$ diblock copolymers of the same chain length as the homopolymers $\left(f=0.5, \alpha_{\mathrm{AB}}=1\right)$ at $\widetilde{\phi}_{\mathrm{HA}}^{0}=0.16$ and $\phi_{\mathrm{AB}}^{0}=0.1$. (a) The total density of A segments $\phi_{\mathrm{A}}$, and (b) the density of $\mathrm{AB}$ diblock copolymers $\phi_{\mathrm{AB}}$ and the densities of individual block $\phi_{\mathrm{CA}}, \phi_{\mathrm{CB}}$. The thin lines in (b) are for $\phi_{\mathrm{CB}}$.

symmetric diblocks in the critical nuclei changes from surfactant-like to co-solvent-like as the blends approach the spinodal from the binodal. Such behavior should be contrasted with the equilibrium behavior, where increasing the immiscibility makes the interface between the HA-rich and HB-rich phase sharper, and thus makes the diblocks behave more like surfactant. ${ }^{6-8}$ Therefore, the local environment of the critical nuclei is important in determining the distribution of diblocks and it is generally not justified to deduce the behavior of the diblocks in the critical nuclei from the equilibrium behavior.

We now return to the main question that we posed in Sec. I, namely, whether the addition of AB diblock copolymers increases or decreases the free energy barrier for nucleation in HA/HB binary blends. The experimentally most relevant situation is a comparison at fixed temperature and fixed ratio of the amount of HA and HB. Thus we first present the result at fixed $\widetilde{\chi}$. Figure 5 shows the free energy barrier as a function of the amount of diblocks added for three values of $\tilde{\chi}$. In this calculation, the degree of polymerization of the

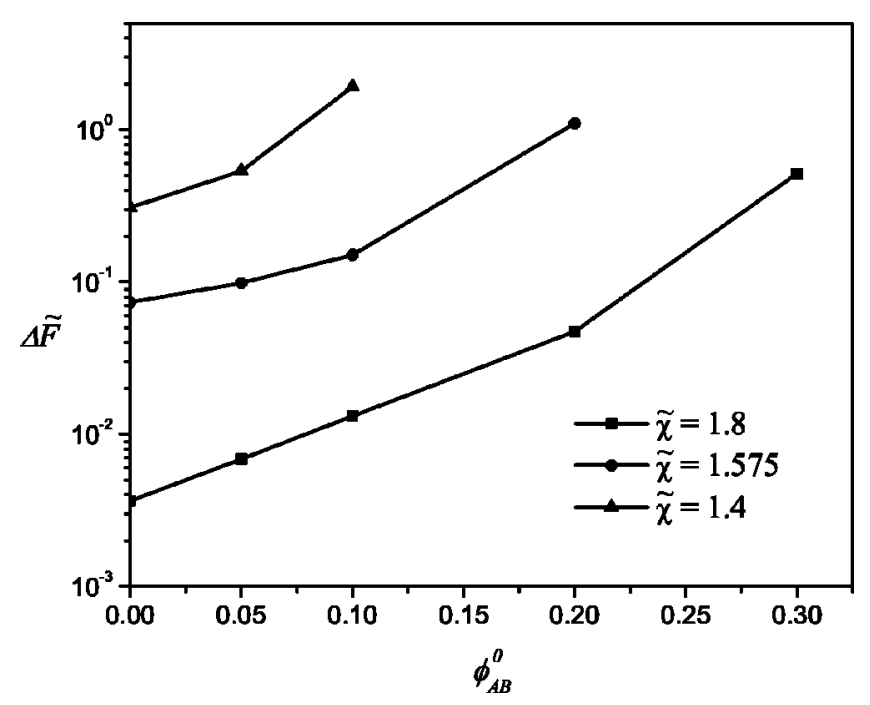

FIG. 5. Free energy barrier for the formation of the critical nuclei as function of the amount of added symmetric diblock copolymers $\left(f=0.5, \alpha_{\mathrm{AB}}\right.$ $=1$ ) at fixed $\widetilde{\phi}_{\mathrm{HA}}^{0}=0.16$ and several values of $\tilde{\chi}$.

diblocks is taken to be the same as that of the homopolymers, i.e., $\alpha_{\mathrm{AB}}=1$. Clearly in all three cases, the free energy barrier increases with increasing amount of diblock copolymers. The closer the system is to the spinodal of the binary system, the stronger is the effect. Because the free energy barrier shown in Fig. 5 is scaled by $N^{1 / 2}$, for long polymers the increase in the actual free energy barrier can be substantial. Therefore in a binary system very close to its spinodal, it is possible to suppress nucleation by the addition of a small amount of $A B$ diblock copolymers. Thus our results indicate that the addition of symmetric $\mathrm{AB}$ diblock copolymers with $\alpha_{\mathrm{AB}}=1$ to $\mathrm{HA} / \mathrm{HB}$ blends not only stablizes the blends thermodynamically but also kinetically by increasing the free energy barrier for nucleation, with the increase driven largely by the decreased thermodynamic driving force. This finding is consistent with the experimental results by Balsara and co-workers, where they find that the addition of diblock copolymers can switch the mechanism of phase separation from spinodal decomposition to nucleation or otherwise decrease the rate of nucleation ${ }^{11,12}$ in the metastable region.

Although the physically relevant comparison for the effects of added diblock copolymers is at fixed $\chi$ (or equivalently $\tilde{\chi})$, it is of theoretical interest to introduce a rescaled $\chi$ using the actual critical $\chi_{c}$ of the ternary system $\tilde{\chi}^{\prime}$ $=\chi^{\prime} \chi_{c}$. In terms of this reduced $\tilde{\chi}^{\prime}$, the coexistence curve is unaffected by the addition of symmetric diblocks while the spinodal boundary $\widetilde{\chi}_{\mathrm{sp}}^{\prime}$ decreases (not shown). Thus at fixed $\tilde{\chi}^{\prime}$, adding symmetric diblocks draws the blends closer to the spinodal. Correspondingly, the free energy barrier decreases.

Finally, if symmetric diblocks are added while keeping the total A/B monomer ratio fixed rather than $\widetilde{\phi}_{\mathrm{HA}}^{0}$, Fig. 6 indicates that the nucleation barrier increases with more symmetric diblocks added at fixed $\tilde{\chi}$. At a fixed total A/B ratio, adding symmetric diblock copolymers shifts both the coexistence and spinodal boundary to lower temperature; thus the blends are farther away from the spinodal and closer to the coexistence. 


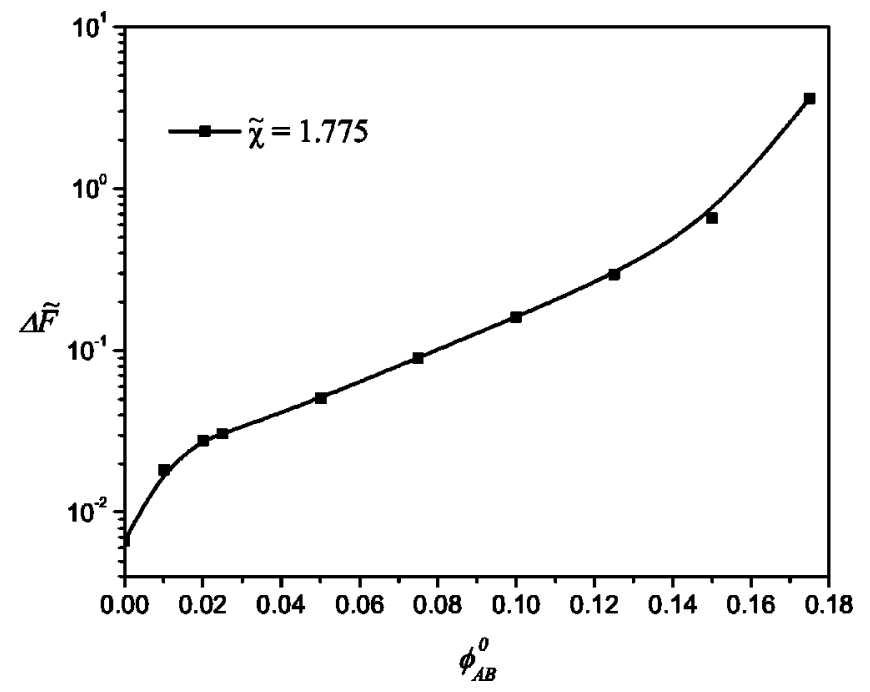

FIG. 6. Free energy barrier for the formation of the critical nuclei as function of the amount of added symmetric diblock copolymers $\left(f=0.5, \alpha_{\mathrm{AB}}\right.$ $=1)$ at fixed total A-segment volume fraction $\phi_{\mathrm{A}}^{0}=0.16$.

These comparisons at different conditions suggest that the qualitative effects of symmetric $\mathrm{AB}$ diblock copolymers on the work of critical nuclei formation can be understood by the shifts in the spinodal and the binodal boundary. If both the spinodal and the binodal boundaries are shifted to higher temperatures, the resulting ternary blends are closer to the spinodal and farther away from the coexistence, and the free energy barrier decreases. On the other hand, if both the spinodal and coexistence are shifted to lower temperatures, the free energy barrier increases. When the shifts of the spinodal and coexistence are in opposite directions, the effects are not obvious, as will be demonstrated later when we discuss the case of asymmetric diblocks.

The radius of the critical nuclei as a function of $\tilde{\chi}$ is also found to be strongly correlated with the thermodynamic state of the ternary system. In Fig. 7, we show the radius of the

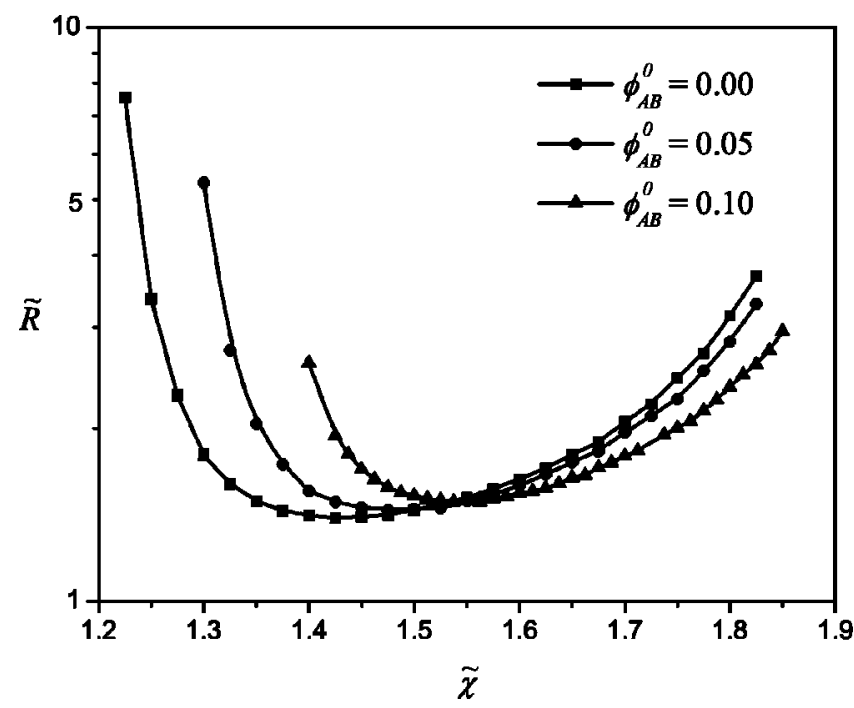

FIG. 7. Radii of the critical nuclei as function of $\tilde{\chi}$ at fixed $\widetilde{\phi}_{\mathrm{HA}}^{0}=0.16$ for several values of the added amount of symmetric $\mathrm{AB}$ diblock copolymers of the same chain length $\left(f=0.5, \alpha_{\mathrm{AB}}=1\right)$.

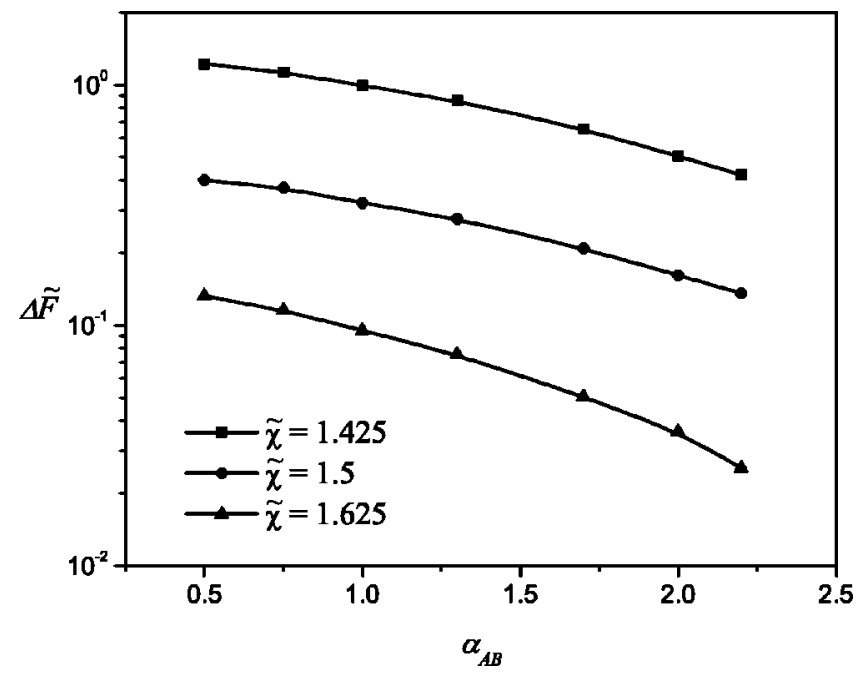

FIG. 8. Free energy barrier for the formation of the critical nuclei as function of the relative chain length of the symmetric $\mathrm{AB}$ diblock copolymers $\left(f=0.5, \phi_{\mathrm{AB}}^{0}=0.1\right)$ at fixed $\widetilde{\phi}_{\mathrm{HA}}^{0}=0.16$ and several values of $\tilde{\chi}$.

critical nucleus as a function of $\tilde{\chi}$ at several values of the amount of symmetric diblocks. Because both the coexistence and the spinodal curves are shifted to larger $\tilde{\chi}$ by adding symmetric block copolymers, and the dimension of the critical nucleus should diverge at both limits, at a fixed $\tilde{\chi}$, the radius of the critical nucleus increases with increasing the amount of block copolymers when close to the coexistence boundary but decreases when close to the spinodal.

The relative degree of polymerization of symmetric block copolymers to that of the homopolymers has effects on their interfacial activity and compatibilizing capability. Both theoretical calculation ${ }^{19}$ and experiment ${ }^{19,20}$ show that the longer block copolymers have stronger interfacial activity and compatibilizing effects for the equilibrium phaseseparated states.

The degree of polymerization of symmetric diblocks also affects the free energy barrier of nucleation. Figure 8 indicates that at the same volume fraction and fixed $\tilde{\chi}$, the free energy barrier decreases with increasing chain length of the symmetric diblock copolymers. This behavior can be rationalized from the phase diagram $\tilde{\chi}$ versus $\alpha_{\mathrm{AB}}$ shown in Fig. 3: increasing the relative chain length of symmetric diblocks while maintaining the same volume fraction has no effect on the binodal boundary, but shifts the spinodal to lower $\tilde{\chi}$. Thus at fixed $\tilde{\chi}$, adding longer symmetric diblocks makes the blends closer to the spinodal boundary, and thus decreases the work of formation of critical nuclei.

The radius of critical nuclei as a function of $\tilde{\chi}$ at different diblock chain lengths (not shown) also can be correlated with the change of phase diagram. With increasing the relative chain length of symmetric block copolymers, the dimension of critical nuclei decreases near the coexistence boundary, while it increases near the spinodal. Near the spinodal, at fixed $\tilde{\chi}$, increasing the relative chain length of symmetric block copolymers makes the blends closer to the spinodal and thus increases the radii of critical nuclei.

Compared to pure binary blends $\mathrm{HA} / \mathrm{HB}$, nucleation in blends added with long enough symmetric diblocks can ex- 


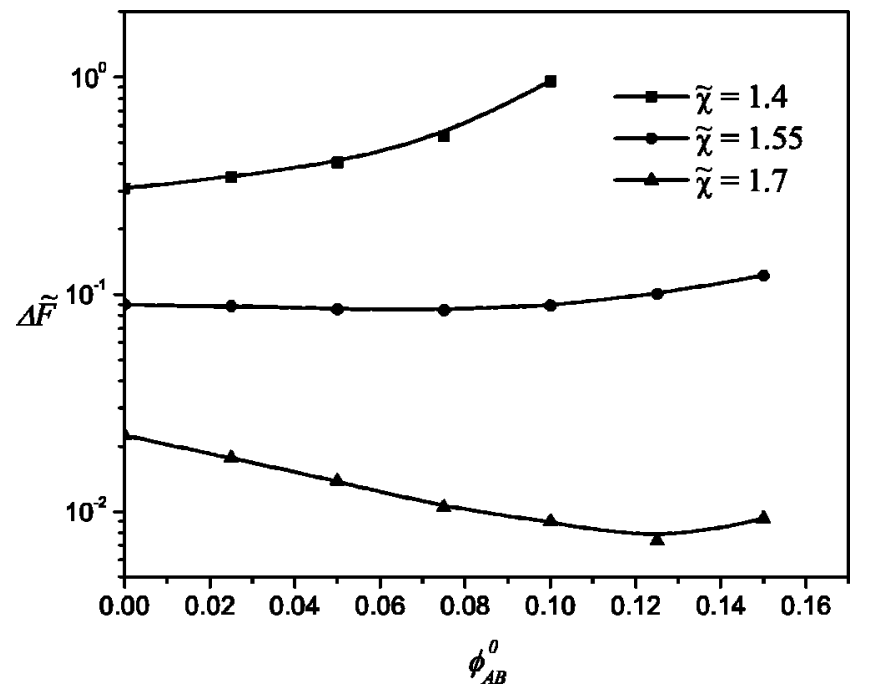

FIG. 9. Free energy barrier for the formation of the critical nuclei as function of the amount of the symmetric $\mathrm{AB}$ diblock copolymers for $\alpha_{\mathrm{AB}}=1.5$ at fixed $\widetilde{\phi}_{\mathrm{HA}}^{0}=0.16$ and several values of $\widetilde{\chi}$.

hibit lower free energy barrier. Figure 9 shows the dependence of the nucleation barrier on adding symmetric diblock copolymers of relative degree of polymerization $\alpha_{\mathrm{AB}}=1.5$ for several values of $\tilde{\chi}$. While at $\tilde{\chi}=1.4$, the addition of the diblocks leads to an increase in the free energy barrier, for the two higher values of $\tilde{\chi}$, the free energy barrier first decreases and then increases with the addition of the copolymers. These behaviors can again be correlated with the changes in the phase diagram (not shown), where the spinodal shows the nonmonotonic change with the amount of the diblocks. At $\tilde{\chi}=1.4$, the addition of the diblocks draws the system closer to the coexistence, while at the other two larger values of $\tilde{\chi}$ with the low addition of the diblocks, the dominant effect is a decreased distance to the spinodal and with the addition of more diblocks, both the spinodal and the coexistence shift toward higher values of $\widetilde{\chi}$ and the blends are nearer to the coexistence.

\section{B. Asymmetric diblock copolymers}

The effects of asymmetric diblock copolymers on the phase diagram are complicated. While the spinodal can be determined analytically according to Eq. (19), the coexistence must be calculated numerically. In Fig. 10, we show the phase diagram of binary blends $\mathrm{HA} / \mathrm{HB}$ added with asymmetric diblock copolymers $\mathrm{AB}$ of the same chain length $\alpha_{\mathrm{AB}}=1.0$ for fixed $\phi_{\mathrm{AB}}^{0}=0.1$. If the phase diagram is represented by $\tilde{\chi}$ versus $f$, the coexistence $\widetilde{\chi}_{\text {co }}$ has a maximum at $f=0.5$, however; the spinodal $\widetilde{\chi}_{\mathrm{sp}}$ has its maximum near $f$ $=0.15$.

The composition of asymmetric diblock copolymers strongly affects the distribution of the diblocks in critical nuclei. While the symmetric diblocks act either as surfactants or co-solvents depending on the proximity to the coexistence or the spinodal, very asymmetric diblocks behave more like the homopolymers and are thus either enriched in or excluded from the critical nuclei, with the extent of enrichment or exclusion decreasing as the spinodal is approached. Thus

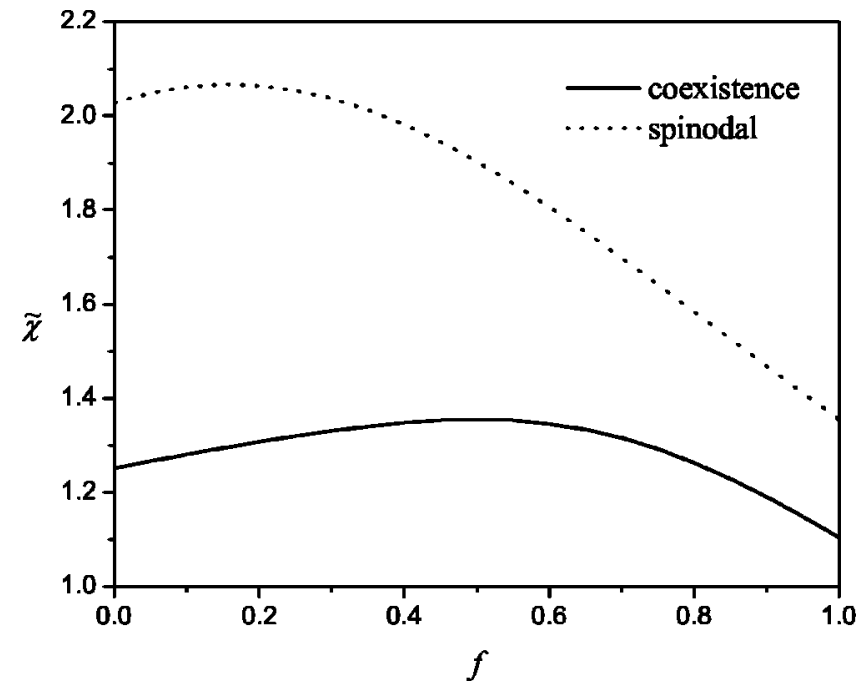

FIG. 10. Phase diagram showing the effect of the composition of the AB diblock copolymers of the same chain length $\left(\alpha_{\mathrm{AB}}=1\right)$ at fixed $\widetilde{\phi}_{\mathrm{HA}}^{0}$ $=0.16$ and $\phi_{\mathrm{AB}}^{0}=0.1$.

the main effects on nucleation for very asymmetric diblocks can be understood as an effective change in the composition of the orginal HA/HB binary blends. For example, at large $f$, the diblocks are more like the HA chains and their addition effectively increases the composition of the HA, and from the inverted parabolic shape of the coexistence and the spinodal curves of the binary system, this change brings the system closer to the spinodal and thus decreases the free energy barrier relative to the pure HA/HB binary system. Away from these limiting cases, however, the behavior of the nucleation barrier with the composition of the diblocks is not simple, as is clear from Fig. 11(a), which reflects the nonsimple dependence of the coexistence and the spinodal with $f$. For example, when $0.15<f<0.5$, increasing the composition of the A block increases the coexistence $\tilde{\chi}_{\text {co }}$ but decreases the spinodal $\tilde{\chi}_{\mathrm{sp}}$.

While the effects of the asymmetry of the diblocks are not simple at the experimentally relevant condition of fixed $\tilde{\chi}$, in terms of fixed $\widetilde{\chi}^{\prime} \equiv \chi^{\prime} \chi_{c}$ where $\chi_{c}$ is the actual critical value of $\chi$ for the ternary system, a relatively simple picture emerges. In a phase diagram $\widetilde{\chi}^{\prime}$ versus $f$ (not shown), both the coexistence and the spinodal boundary decreases monotonically with increasing the composition of the A block. Therefore, as shown in Fig. 11(b), at fixed $\widetilde{\chi}^{\prime}$, increasing the composition of A block lowers the free energy barrier of nucleation monotonically.

The dependence of the size of critical nucleus on $\tilde{\chi}$ at different $f$ reflects changes in the phase diagram. Relative to the symmetric case of $f=0.5$, both the coexistence and the spinodal shift to lower $\widetilde{\chi}$ in the case of $f=0.75$. Correspondingly, the size of critical nucleus for $f=0.75$ is smaller near the coexistence but larger near the spinodal than that for $f$ $=0.5$, as shown in Fig. 12. For $f=0.25$, the coexistence boundary shifts to smaller $\tilde{\chi}$ while the spinodal shifts to larger $\tilde{\chi}$ relative to the blends with symmetric block copolymers. Consequently, the blend with $f=0.25$ has smaller critical nuclei than that with $f=0.5$ near both the spinodal and the coexistence. 

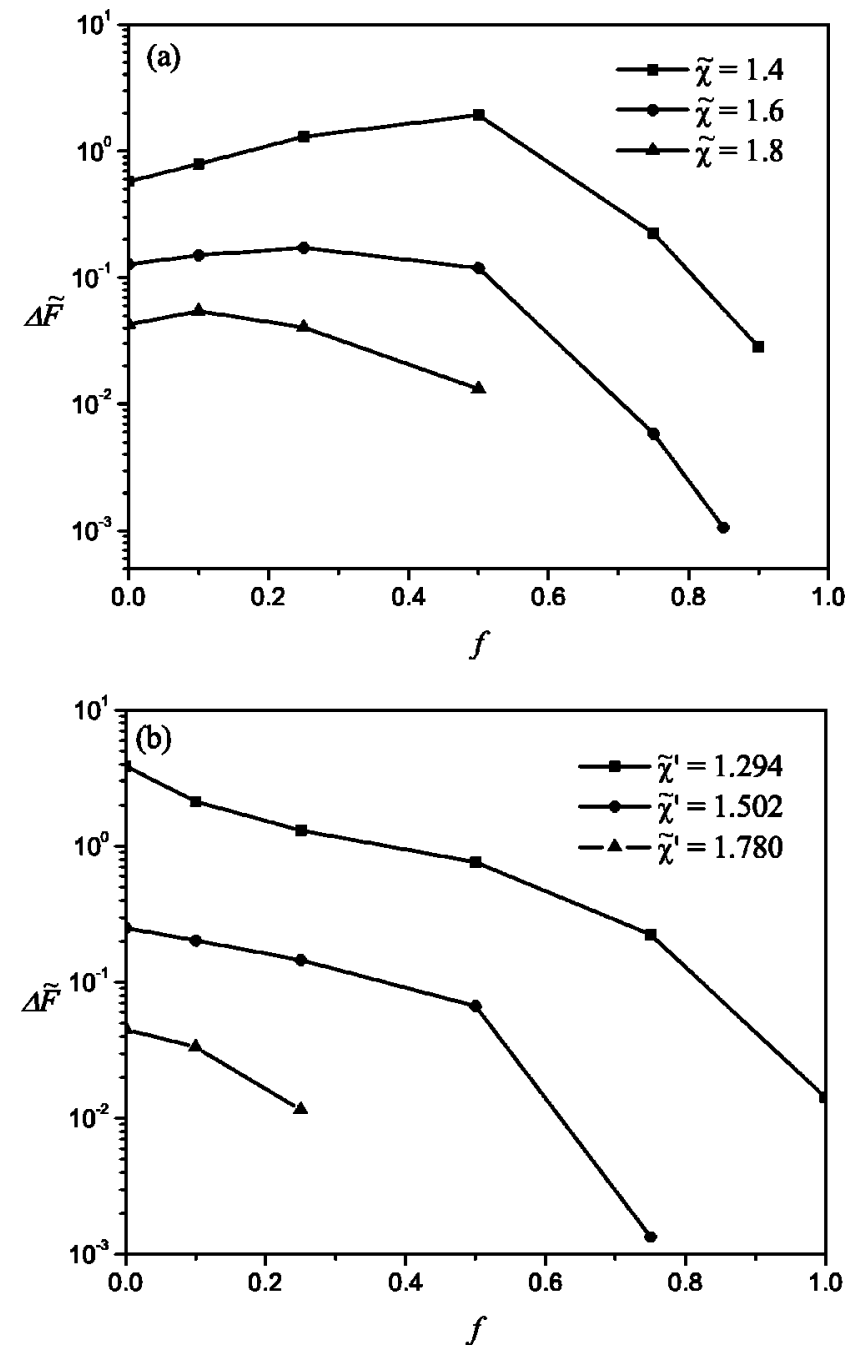

FIG. 11. Free energy barrier for the formation of the critical nuclei as function of the composition of the $\mathrm{AB}$ diblock copolymers of the same chain length $\left(\alpha_{\mathrm{AB}}=1\right)$ at fixed $\widetilde{\phi}_{\mathrm{HA}}^{0}=0.16$ and $\phi_{\mathrm{AB}}^{0}=0.1$. In (a) $\tilde{\chi}$ is kept constant; in (b) $\tilde{\chi}^{\prime}$ is kept constant.

\section{CONCLUSIONS}

Using the self-consistent field method, we have examined the effects of adding diblock copolymers $\mathrm{AB}$ on nucleation in binary $\mathrm{HA} / \mathrm{HB}$ blends, with an emphasis on the changes in the nucleation barrier. Upon the addition of diblock copolymers at a fixed ratio of the amount of the two homopolymers, the free energy barrier can either increase or decrease relative to the pure $\mathrm{HA} / \mathrm{HB}$ blends, depending on the degree of polymerization and composition of the diblocks, as well as the location of the system in the metastable part of the phase diagram. The qualitative trend can be deduced from the shift in the coexistence boundary and the spinodal. If the addition of the copolymers shifts both the coexistence and the spinodal to lower temperature (or higher $\chi$ ), the free energy barrier increases relative to that in the pure binary blends. If the addition of the copolymers shifts both the coexistence and the spinodal to higher temperature (or lower $\chi$ ), the free energy barrier decreases. The behavior becomes complicated when the shifts in the coexistence and the spinodal are in the oppositive directions.

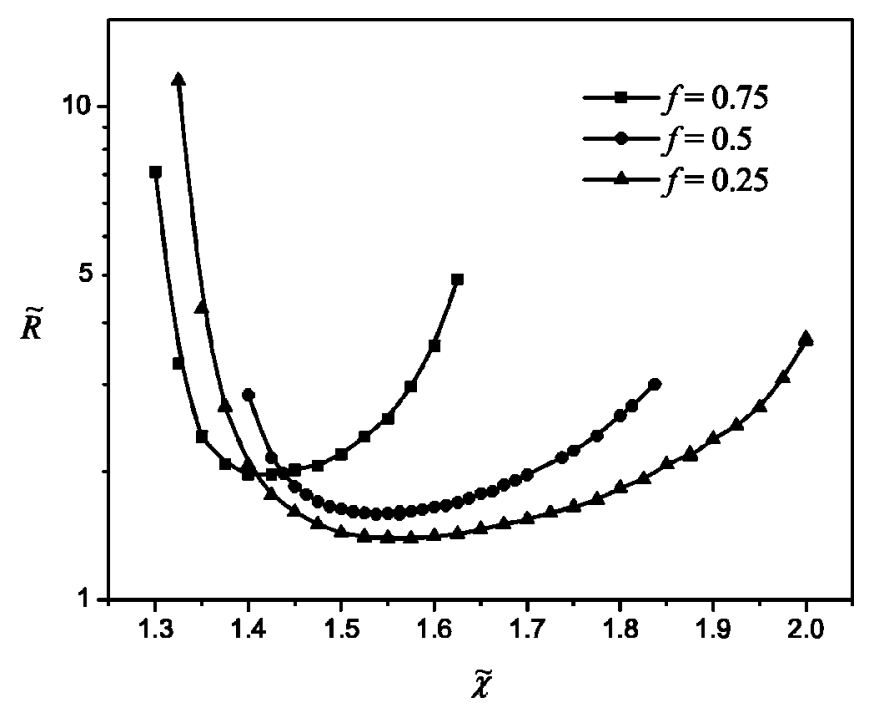

FIG. 12. Radii of the critical nuclei as function of $\tilde{\chi}$ at fixed $\widetilde{\phi}_{\mathrm{HA}}^{0}=0.16$ and $\phi_{\mathrm{AB}}^{0}=0.1$, showing the effect of composition asymmetry in the diblock copolymers of the same length as the homopolymers $\left(\alpha_{\mathrm{AB}}=1\right)$.

The distribution of diblock copolymers in the critical nuclei depends on the composition of the diblocks and the quench depth. Near the coexistence, symmetric diblocks exhibit surfactant behavior, residing preferentially at the interface of the critical nuclei. Near the spinodal, they act more like co-solvent with a relatively uniform distribution. Such behavior is in contrast to the equilibrium behavior, where increasing the immiscibility makes the interface between the HA-rich and HB-rich phase sharper, and thus makes the diblocks behave more like surfactant. ${ }^{6-8}$

Our work in this paper has been focused on nucleation leading to macroscopic phase separation. However, the phase behavior of the full $\mathrm{AB} / \mathrm{HA} / \mathrm{HB}$ ternary system is extremely rich and complex. While straightforward application of the self-consistent field methods can successfully treat the periodic and disordered phases, it is unable to treat emulsion droplets and swollen micelles. Because these structures can be considered as arising from large but local concentration fluctuations, similar to nucleation leading to macroscopic phase separation, the method we have developed for nucleation can be easily extended to study the thermodynamics of these structures as well as the nucleation event that leads to their formation. We plan to do so in future work.

\section{ACKNOWLEDGMENTS}

Financial support by the NSF of China and the Special Project for Fundamental Researches of the Ministry of Science and Technology is acknowledged. Z.G.W. acknowledges support by the US National Science Foundation (DMR-9970589).

${ }^{1}$ S. M. Wood and Z.-G. Wang, J. Chem. Phys. 116, 2289 (2002).

${ }^{2}$ S. T. Milner and H. W. Xi, J. Rheol. 40, 663 (1996).

${ }^{3}$ R. A. L. Jones, Curr. Opin. Solid State Mater. Sci. 2, 673 (1997).

${ }^{4}$ W. C. Hu, J. T. Koberstein, J. P. Lingelser, and Y. Gallot, Macromolecules 28, 5209 (1995).

${ }^{5}$ R. Holyst and M. Schick, J. Chem. Phys. 96, 7728 (1992).

${ }^{6}$ D. Broseta and G. H. Fredrickson, J. Chem. Phys. 93, 2927 (1990). 
${ }^{7}$ M. W. Matsen and M. Schick, Macromolecules 27, 187 (1994).

${ }^{8}$ F. S. Bates, W. W. Maurer, P. M. Lipic, M. A. Hillmyer, K. Almdal, K. Mortensen, G. H. Fredrickson, and T. P. Lodge, Phys. Rev. Lett. 79, 849 (1997).

${ }^{9}$ H. S. Jeon, J. H. Lee, N. P. Balsara, and M. C. Newstein, Macromolecules 31, 3340 (1998)

${ }^{10}$ H. S. Jeon, J. H. Lee, and N. P. Balsara, Macromolecules 31, 3328 (1998).

${ }^{11}$ N. P. Balsara, C. Lin, and B. Hammouda, Phys. Rev. Lett. 77, 3847 (1996).

${ }^{12}$ A. A. Lefebvre, J. H. Lee, H. S. Jeon, N. P. Balsara, and B. Hammouda, J. Chem. Phys. 111, 6082 (1999).

${ }^{13}$ V. Talanquer and D. W. Oxtoby, J. Chem. Phys. 106, 3673 (1997).
${ }^{14}$ V. Talanquer and D. W. Oxtoby, J. Chem. Phys. 113, 7013 (2000).

${ }^{15}$ N. A. M. Besseling and M. A. Cohen Stuart, J. Chem. Phys. 110, 5432 (1999).

${ }^{16}$ R. B. Thompson and M. W. Matsen, J. Chem. Phys. 112, 6863 (2000).

${ }^{17}$ P. K. Janert and M. Schick, Macromolecules 30, 137 (1997).

${ }^{18}$ C. Huang, M. Olvera de la Cruz, and B. W. Swift, Macromolecules 28, 7996 (1995).

${ }^{19}$ R. Israels, D. Jasnow, A. C. Balazs, L. Guo, G. Krausch, J. Sokolov, and M. Rafailovich, J. Chem. Phys. 102, 8149 (1995).

${ }^{20}$ S. H. Anastasiadis, R. Gancarz, and J. B. Koberstein, Macromolecules 22, 1449 (1989) 